\title{
Electromagnetic Crosstalk Penalty in 2.5GB/s and 10GB/S Serial optical modules
}

\author{
Xiaomin Jin, Fei Wang *, Keith D. Lystad, and Musoke H. Sendaula** \\ * Xiaomin Jin and Fei Wang are with Electrical Engineering Department, California Polytechnic State University, San Luis

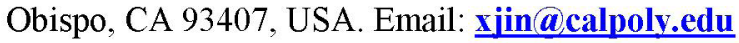 \\ ** Keith D. Lystad, and Musoke H. Sendaula are with Department of Electrical and Computer Engineering, Temple University, \\ Philadelphia, PA19122, USA
}

\begin{abstract}
Electromagnetic crosstalk poses a serious problem in advanced serial communication modules. This paper focuses on the primary sources of crosstalk penalty in a $2.5 \mathrm{~Gb} / \mathrm{s}$ optic transceiver and a $10 \mathrm{~Gb} / \mathrm{s}$ optic transponder. A novel method to quantify the crosstalk penalty by observing a receiver's bit-error-ratio (BER) versus transmitter to receiver signal phase is proposed. A coupled microstrip transmission line model is also explored to demonstrate inductive crosstalk coupling.
\end{abstract}

\section{Introduction}

As the industry advances in circuit and assembly technologies, module sizes are shrinking; concurrently, receiver dynamic range is expanding to be competitive in the communication component marketplace. The resulting outcomes are that receivers are becoming increasingly more sensitive while the transmitter and receiver are being packaged in closer proximity, increasing the risk of experiencing crosstalk penalty. For the purpose of this study, crosstalk penalty is defined as receiver sensitivity degradation due to electromagnetic susceptibility to conducted and/or emitted transmitter radiation. The crosstalk between two channels is defined as the ratio of the output channel A (with no input signal) divided by the output of channel B (excited by input signal). [1]-[3]

$$
\text { Crosstalk }=20 \log \frac{\left|v_{O A}\right|}{\left|v_{O B}\right|} d B
$$

In this paper, channel A would represent the output of the receiver pre-amplifier and channel $B$ would be the output of the transmitter driver, as depicted in Figure 1. The crosstalk measurement is a very difficult task in fiber-optic module due to the nature of the signals that are involved. It is not always feasible to directly measure crosstalk, which requires direct access to the involved interconnects. [4] [5] A method for quantifying crosstalk in terms of relative receiver BER penalty is proposed in this paper, mostly using equipment that would be standard in a well-equipped communication module development lab. A similar approach is undertaken in two different applications: a $2.5 \mathrm{~Gb} / \mathrm{s}$ fiber optic transceiver and a $10 \mathrm{~Gb} / \mathrm{s}$ fiber optic transponder. These applications were chosen as cross-sections of high-speed communication modules that are available today. They do not represent the entire population, but may serve as a basis of measurement methods that can be easily modified for similar modules.

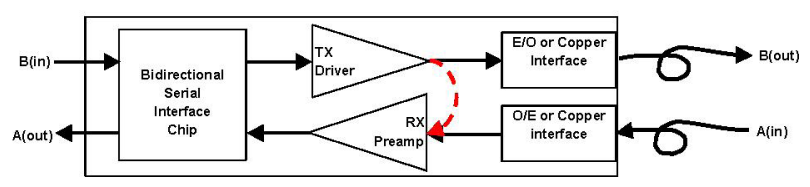

Fig. 1. Simplified bidirectional communication module, depicting crosstalk from transmitter (Channel B) to receiver (Channel A).

\section{Experiment results}

Figure 2 shows the experimental setup for crosstalk penalty measurement of a $2.5 \mathrm{~Gb} / \mathrm{s}$ serial fiber optic transceiver. The device-under-test (DUT) is a pluggable device. The input to the device's optical receiver is a $1310 \mathrm{~nm}$ laser source modulated by a $2.488 \mathrm{~GB} / \mathrm{s}$ pattern generator. The generated pattern is a pseudo-random bit stream (PRBS) with a seed of $2^{31}-1$. The laser source is fed to an adjustable optical attenuator with a power tap to measure the power to the DUT. The receive path of the transceiver contains an avalanche photodiode (APD) to create an output current proportional to photon illumination, a trans-impedance amplifier (TIA) to convert the current to an analog voltage, and a limiting amplifier (LIA) to create a digital output from the analog voltage. The output is differential, where the non-inverting signal is sent into a clock and data recovery (CDR) unit, which derives clock and data for the error detector. The 
inverting output is fed to a phase delay element. The variably delayed signal is then fed into the transceiver's transmitter. The phase relationship between transmitter and receiver can then be adjusted into maximize or minimize the crosstalk penalty, i.e. the bit error ratio (BER) at the error detector.

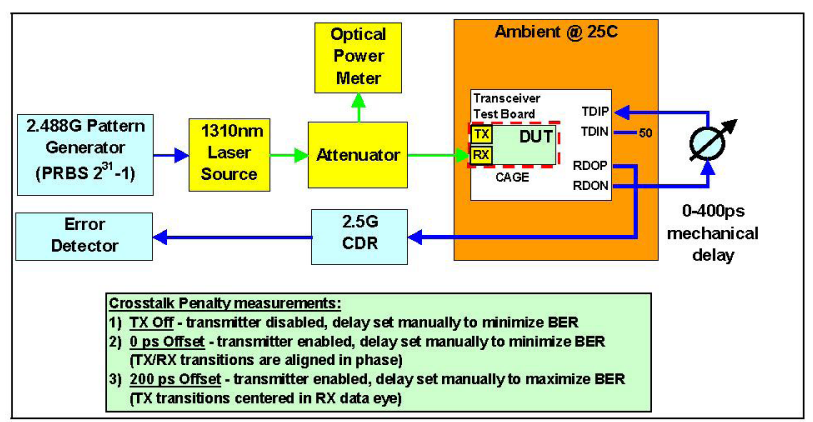

Fig. 2. Setup diagram for testing crosstalk penalty of a $2.5 \mathrm{~GB} / \mathrm{s}$ serial fiber optic transceiver.

The sensitivity of the transceiver's receiver was obtained by adjusting the optical attenuation in $0.5 \mathrm{~dB}$ increments and measuring the resulting BER. To measure the transmitter to receiver crosstalk penalty, three sensitivity curves were measured as shown and described in Figure 3. In this particular device, the best sensitivity of $-31 \mathrm{dBm}$ was measured when the transmitter was disabled. The best-case crosstalk penalty of $0.3 \mathrm{~dB}$ was observed when the transmitter transitions were phase-aligned with the receive transitions. The worst-case penalty of $1.3 \mathrm{~dB}$ was observed when the transmit transitions were in the center of the receive data eye, or $200 \mathrm{ps}$ offset for a $2.5 \mathrm{~Gb} / \mathrm{s}$ signal ( $400 \mathrm{ps}$ bit period).

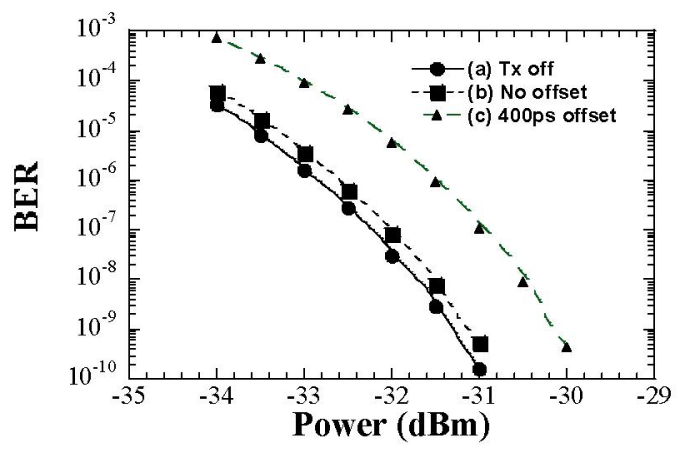

Fig. 3. Sensitivity penalty measurement (BER) of a $2.5 \mathrm{~GB} / \mathrm{s}, 40 \mathrm{~km}$ serial optical transceiver with a) transmit data disabled b) transmit data enabled with transitions phase-aligned with the receiver and c) transmit data enabled with 0.5 UI (400ps) phase offset from the receiver.

The block diagram of the crosstalk test setup for a $10 \mathrm{~Gb} / \mathrm{s}$ fiber optic transponder is shown in Figure 4. Although these devices are not fully serial, they contain a serial transmitter and receiver pair for transmission over optical fiber. The serial transmitter and receiver optical engines are respectively mated with a 16:1 multiplexer and a 1:16 demultiplexer. The setup is similar to that of the $2.5 \mathrm{~Gb} / \mathrm{s}$ transceiver with some notable exceptions: the line rate is at 10 gigabits per second, the data on the electrical module interface is parallel at $1 / 16$ of the line rate, and reference clocks are required for data recovery and retiming. The data originates from a PRBS $2^{31}-1$ pattern generator at $9.95328 \mathrm{~GB} / \mathrm{s}$ (OC-192 SONET rate), which drives a $1550 \mathrm{~nm}$ laser transmitter. The optical data is then sent to an optical attenuator with a calibrated power tap for monitoring the input level to the transponder under test. The receiving path of the transponder uses a reference clock to perform clock and data recovery, then it de-multiplexes the serial data into 16 parallel channels. The parallel data is electrically looped back with a parallel data clock to the transmitter path multiplexer, through an external multiplexer/demultiplexer pair on the evaluation board. The reference clock for the transmitter, which retimes the data for serial transmission, is a delayed copy of the receive path recovered clock; a data FIFO enables the transmitter parallel clock and serial clock to be different. The phase relationship of the serial transmitted data to the serial received data can then be manually adjusted for the crosstalk measurement. The Bit Error Rate Tester (BERT) is driven directly from an external 16:1 multiplexer on the receiving path of the test set, allowing the bit error ratio of the receiver to be measured independently from the transmitter while experiencing crosstalk from the transmitting path. The resulting BER variation as the serial transmit delay is swept from 0 to $100 \mathrm{ps}$ in relation to the serial receive data is shown in Figure 5. The measurement was taken at $25 \mathrm{C}$ and an elevated temperature of $70 \mathrm{C}$ with similar results; the BER variation is approximately one half decade at an error ratio of 1e-4 ( 1 error per 10,000 bits). This BER variation translates to approximately $0.3 \mathrm{~dB}$ sensitivity penalty. 


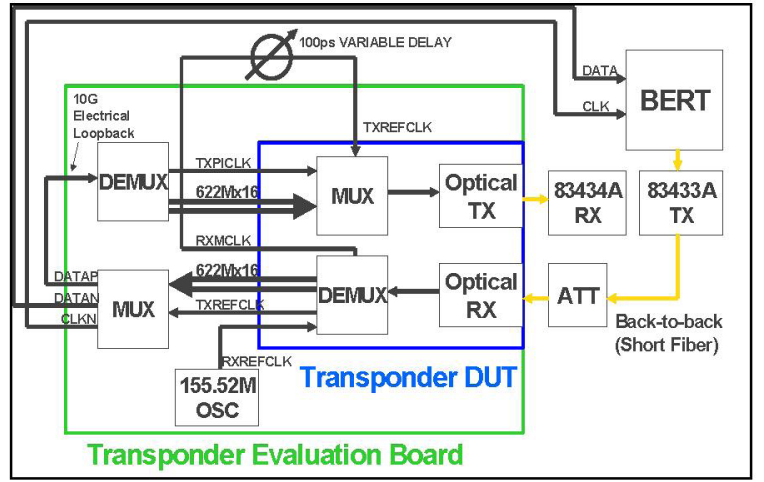

Fig. 4 The setup diagram for testing crosstalk penalty of a $10 \mathrm{~GB} / \mathrm{s}$ multiplexing/de-multiplexing fiber optic transponder.

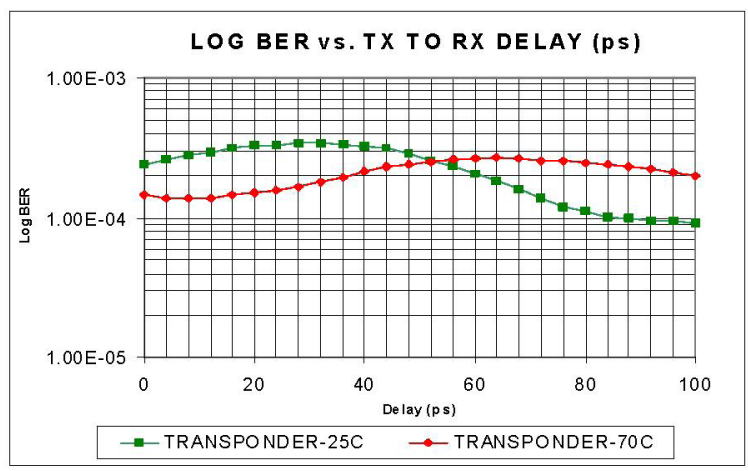

Fig. 5 Bit error ratio variation of a $10 \mathrm{~GB} / \mathrm{s}$ multiplexing / de-multiplexing fiber optic transponder as the transmitter to receiver delay is varied by one unit interval (100ps), measured at $25 \mathrm{C}$ and $70 \mathrm{C}$.

\section{Coupled Microstrip Crosstalk Simulation}

Slots in the ground and other metallization planes are common features in densely packaged PCBs and multi-level on-chip integrated circuits, often caused by vias and thru holes. This diverts the ground plane return current, which can cause undesirable effects such as crosstalk, EMI noise and impedance changes. In highspeed circuits, current flows in the path of least inductance; in a microstrip line, current in the ground plane tends to flow directly under the signal trace. A slot in the ground plane causes the return current to take a circuitous route around the slot, which translates into increased inductance. The increased area of the return current loop causes EMI emissions that are directly proportional to the loop area.

To simulate our bidirectional communication module, we use a bidirectional micro-strip transmission pair, the structure is shown in Fig. 6. The structure to be simulated was kept simple for two main reasons: first, the Sonnet Lite 9.51 EM simulator has limited capability; and second, crosstalk analysis complexity would increase exponentially if more entities were included. We simulate a bidirectional transmission, from Port 1 to Port 2 and from Port 3 to Port 4 (two blue lines). One case has a solid ground plane. The other has a 10-mil cleave in the ground plane underneath.

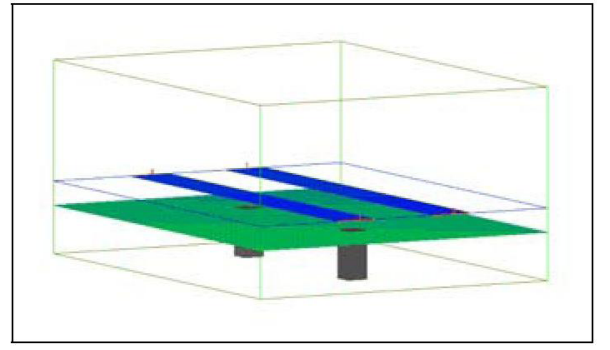

(a)

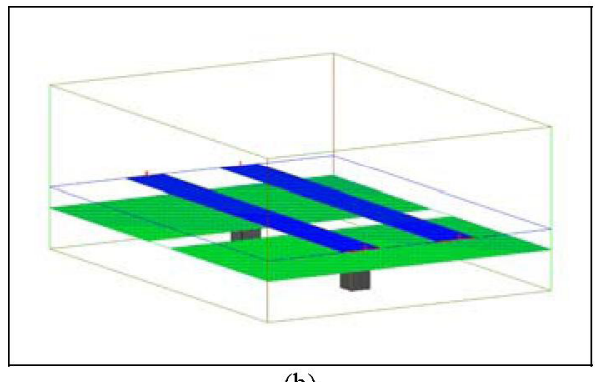

(b)

Fig. 6 (a) 3-D view of a bidirectional microstrip transmission pair on a 10-mil thick bismaleimide triazine (BT) substrate, with a solid ground plane underneath. The ground plane is suspended above absolute ground and connected by vias to enable cleaving of the ground plane. (b) 3-D view of a bidirectional microstrip transmission pair on a 10-mil thick BT substrate, with a 10-mil cleave in the ground plane underneath.

Fig. 7 shows the field comparison of solid ground plan and the cleaved ground plan. Our calculation shows that a cleave in microstrip ground was found to have a profound effect on port isolation, which would become visible as sensitivity penalty within a communication module. Crosstalk is inevitable within 
any enclosure with bidirectional transmission, but by employing good RF design practice the penalty caused by crosstalk noise can be substantially reduced. [6] [7] As technology advances enable smaller and faster electronic devices, more emphasis must be placed on designing for crosstalk isolation to maintain the high level of signal integrity that will be required by future advanced communication systems.

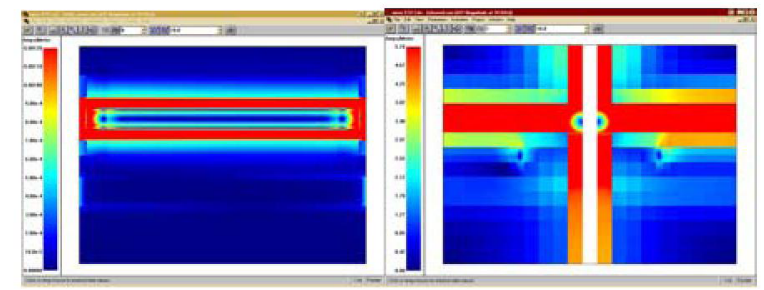

(a)

(b)

Fig. 7 (a) Microstrip interconnect electric field at $10 \mathrm{GHz}$ with a solid ground plane, Port 1 to 2 active. Field was obtained by placing a high impedance sense layer 0.1 mils above the surface. There is minimal fringing to the trace from Port 3 to 4. (b) Microstrip ground plane return current density with a cleaved ground plane, Port 1 active. There is large inductive coupling to the neighboring trace from Port 3 to 4 since return current is forced around the cleave.

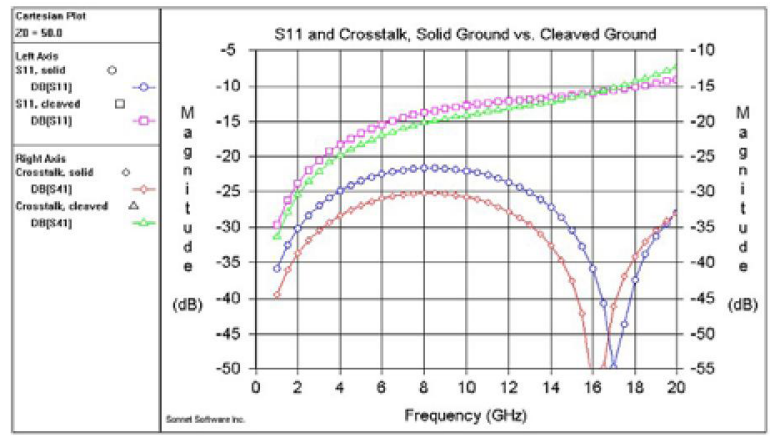

Fig. 8 Return loss (S11) and isolation (S41) of the bidirectional microstrip transmission pair with Port 1 active, with solid and cleaved ground planes. Greater than $10 \mathrm{~dB}$ crosstalk penalty is experienced at $10 \mathrm{GHz}$ due to the ground plane cleave.

Finally, the Return loss was calculated, which shows how well the characteristic impedance of the interconnect matches that of the driver, in this case $\mathbf{5 0}$ $\mathrm{ohm}$. Return loss of negative infinity would be ideal, which indicates zero reflection. As seen in Figure 8, the return loss (S11) of the microstrip interconnect with a solid ground plane is $-22 \mathrm{~dB}$ at $10 \mathrm{GHz}$. The return loss in discontinuous ground plane case degrades to $-13 \mathrm{~dB}$ at $10 \mathrm{GHz}$ due to the impedance mismatch from the ground plane discontinuity, where the transmission line looks more inductive. Crosstalk is the amount of coupling from port 1 (active trace driver) to port 4 (inactive trace receiver), designated by $\mathrm{S} 41$ in terms of scattering parameters. The coupling from port 1 to port 4 increases from $-30 \mathrm{~dB}$ to $-20 \mathrm{~dB}$ at $10 \mathrm{GHz}$ due to electromagnetic radiation from the ground plane discontinuity.

\section{Summary}

Electromagnetic crosstalk in bidirectional serial communication modules is a complex and elusive phenomenon that is not easy to be quantified. Direct measurement of crosstalk in the time domain or frequency domain by probing is not always possible within a module, and removal of the packaging would change the environmental conditions. The crosstalk measurement method proposed in this paper allows the module to be tested as a complete entity, in its ambient operating condition. This provides an accurate and realistic measurement of the maximum crosstalk penalty that would be expected in a real application. A simplified crosstalk model was presented to demonstrate inductive coupling of a bidirectional microstrip transmission pair.

\section{References}

[1] A. Vittal, L. H. Chen, M. Marek-Sadowska, K.Wang, and S. Yang, IEEE Transactions on Computer-Aided Design of Integrated Circuits and Systems, 18, p. 1817, (1999).

[2] H. Johnson and M. Graham, High-Speed Digital Design: A Handbook of Black Magic. Prentice Hall, p. 189, (1993).

[3] R.F. Drayton, R.M. Henderson and L.P.B. Katehi, IEEE Transactions on Microwave Theory and Techniques, 46, p. 900, (1998).

[4] K.M. Coperich, J. Morsey, V.I. Okhmatovski, A.C. Cangellaris and A.E. Ruehli, IEEE Transactions on Microwave Theory and Techniques, 49, p. 1677, (2001).

[5] D.E. Bockelman and W.R. Eisenstadt, IEEE Transactions on Microwave Theory and Techniques, 48, p. 1410, (2000).

[6] L.G. Maloratsky, Microwaves and RF, p.79. (2000).

[7] J.H. Wu, J. Scholvin, J.A. del Alamo and K.A. Jenkins, IEEE Microwave and Wireless Components Letters, 11, p. 410, (2001). 